\title{
Maternal dietary patterns and risk of gestational diabetes mellitus in twin pregnancies: a longitudinal twin pregnancies birth cohort study
}

Li Wen ${ }^{1,2,3 \dagger}{ }^{1}$, Huisheng Ge ${ }^{4 \dagger}$, Juan Qiao ${ }^{1,2,3}$, Lan Zhang ${ }^{1,2,3}$, Xuyang Chen ${ }^{1,2,3}$, Mark D. Kilby, ${ }^{5,6}$, Ying Zhou ${ }^{1,2,3}$, Jie Gan ${ }^{1,2,3}$, Richard Saffery ${ }^{7}$, Jianying Yan ${ }^{8}$, Chao Tong ${ }^{1,2,3^{*}}$ (D) Hongbo Qi ${ }^{1,2,3,8^{*}}$ and Philip N. Baker ${ }^{9}$

\begin{abstract}
Background: Gestational diabetes mellitus (GDM) is correlated with an increased risk of adverse perinatal outcomes for both the mother and offspring. Previous research has reported correlations between maternal dietary patterns and GDM, but such evidence for twin pregnancies is lacking. This study aimed to identify maternal dietary patterns in the second trimester and investigate their relationships with the risk of GDM among women who were pregnant with twins in China.

Methods: A longitudinal twin pregnancies birth cohort study of women who were pregnant with twins in China was conducted. Maternal dietary intake in the second trimester was recorded by using a food frequency questionnaire prior to the diagnosis of GDM among participants from the prospective twin pregnancies birth cohort in Chongqing City. GDM was diagnosed with a $75 \mathrm{~g}$ 2-h oral glucose tolerance test at 23-26 weeks of gestation. Dietary patterns were identified by principal components analysis, and the correlations between dietary pattern and GDM were examined using multivariable logistic regression analyses.
\end{abstract}

Results: Of the 324 participants, 101 (31.2\%) were diagnosed with GDM. Four dietary patterns were identified: a vegetablebased pattern, a poultry-and-fruit-based pattern, a sweet-based pattern and a plant-protein-based pattern. Multivariate analysis showed that none of the dietary patterns were correlated with the risk of GDM among women who were pregnant with twins, but the sweet-based dietary pattern, which was associated with a higher GDM risk for quartile 4 versus quartile 1 (OR 2.69; 95\% Cl: 1.09, 6.66) among non-overweight women (prepregnancy BMl < 24.0).

Conclusion: Dietary patterns were not correlated with later GDM risk among women who were pregnant with twins in western China, whereas a high intake of sweets was associated with a higher risk for GDM among women who were not overweight prior to pregnancy.

Trial registration: ChiCTR-OOC-16008203. Retrospectively registered on 1 April 2016.

Keywords: Dietary patterns, Gestational diabetes, Twin pregnancies, China

\footnotetext{
* Correspondence: chaotongcamu@163.com; qihongbo728@163.com

+ Li Wen and Hui-sheng Ge contributed equally to this work.

'Department of Obstetrics, The First Affiliated Hospital of Chongqing Medical

University, Chongqing 400016, China

Full list of author information is available at the end of the article
}

C The Author(s). 2020 Open Access This article is distributed under the terms of the Creative Commons Attribution 4.0 International License (http://creativecommons.org/licenses/by/4.0/), which permits unrestricted use, distribution, and reproduction in any medium, provided you give appropriate credit to the original author(s) and the source, provide a link to the Creative Commons license, and indicate if changes were made. The Creative Commons Public Domain Dedication waiver (http://creativecommons.org/publicdomain/zero/1.0/) applies to the data made available in this article, unless otherwise stated. 


\section{Introduction}

Gestational diabetes mellitus (GDM) is one of the most common pregnancy complications in which women present with impaired glucose tolerance with an onset or first recognition during pregnancy $[1,2]$. The prevalence of GDM varies from 9.8 to $25.5 \%$ according to the latest diagnostic criteria established by the International Association of Diabetes and Pregnancy Study Groups (IADPSG) in 2010 [3]. A previous study based on a large population suggested that the morbidity rate of GDM in the Chinese population varied from 17.5 to $18.9 \%$ according to the IADPSG criteria [4]. GDM is correlated with adverse effects on mothers and their offspring, such as macrosomia and cesarean section [5]. Although the blood glucose level of GDM patients usually returns to normal within 6 weeks after delivery, GDM increases the risk of postpartum type 2 diabetes among mothers and the risks of obesity or other metabolic complications among the offspring in their later life [6].

Given the known and potential adverse effects of GDM, the identification of risk factors for GDM is warranted. Accumulating evidence has revealed that dietary intake during pregnancy is involved in the development of GDM. High consumption of saturated fat, carbohydrates or animal protein is associated with a higher risk of GDM [710], while polyunsaturated fat intake appears to lower GDM risk [11], but debate on this topic remains [12]. To determine the correlation between food intake and perinatal outcomes, dietary pattern analyses are preferred with the advantages of accounting for food consumption over a given period and taking into account nutrients consumed in combination. For example, a systematic review suggested that vegetarian- or Mediterranean-style dietary patterns reduce the risk of GDM [13], whereas dietary patterns characterized by high intakes of red and processed meat and refined grains are associated with an increased risk of GDM $[14,15]$.

An increasing proportion of twin pregnancies has been observed worldwide in recent decades. Since women pregnant with twins are believed to undergo more complicated physiological changes and have a higher risk of adverse obstetric outcomes when compared to those with singleton pregnancies [16], it is essential to pay close attention to maternal and fetal health in the context of twin pregnancies. Previous studies have reported that twin gestation is associated with an increased risk of GDM [17, 18], and GDM was associated with a higher risk of gestational hypertension and preeclampsia in twin pregnancies [19]. Therefore, the influence of dietary intake on GDM in the context of twin pregnancies is worth exploring.

To date, studies on the effects of dietary habits on GDM development in the context of twin pregnancies are extremely limited. Therefore, the objective of this study is to identify maternal dietary patterns during pregnancy and investigate whether maternal dietary patterns are associated with the risk of developing GDM in the context of twin pregnancies in a Chinese prospective cohort.

\section{Methods \\ Study design and participants}

The current study was conducted with women pregnant with twins in the Chongqing Longitudinal Twin Study (LoTiS) (ChiCTR-OOC-16008203) - the world's largest prospective twin pregnancies birth cohort that was established in Chongqing, China in 2016 with the main aim of elucidating the complex interplay between early-life environmental and genetic risk factors in the context of health and diseases [20]. Study participants were recruited at 1116 weeks' gestation from the First Affiliated Hospital of Chongqing Medical University and Chongqing Women and Children's Health Center between January 2016 and September 2018. Four follow-up clinic visits were carried out throughout pregnancy, and eight pediatric follow-up visits were conducted 3 years after birth. The LoTiS study was approved by the Ethics Committee of the First Affiliated Hospital of Chongqing Medical University (No.201530). Written informed consent was obtained from all participants. Participants were subjected to a $75 \mathrm{~g}$ oral glucose tolerance test (OGTT) between the 23rd and 26th weeks of gestation, and those who completed a food frequency questionnaire prior to the diagnosis of GDM on the same day were eligible for this study.

\section{Dietary assessment}

The maternal average dietary intake over the past 3 months was assessed using a food frequency questionnaire (FFQ) and was recorded correctly by a trained researcher in a face-to-face interview. The FFQ originated from Singapore and has been validated in a singleton pregnancy study conducted in our laboratory [21]. The FFQ consists of 93 specified food items as well as 15 additional questions about dietary behaviors. The participants were asked to recall food intake frequencies (how many times per day or per week or per month) and estimate the food intake portion each time each food item listed was consumed. A photo booklet was shown to participants to help them understand the standard portion sizes. The quantities and frequencies were recorded in detail. The dietary information of individuals was entered electronically for further calculation.

We calculated the daily food intake by averaging the consumption frequency of each food item per day and adjusting the daily food intake for energy intake based on the China Food Composition Database. Some food items were combined into one group of items with similar nutrient profiles or culinary uses. Forty non-overlapping food groups served as the main dataset for investigation. The total frequency of the intake of items in one food group was the sum of all food items consumed in the group. 


\section{Diagnosis of gestational diabetes mellitus}

GDM was diagnosed by a $75 \mathrm{~g} 2$-h OGTT only when the following plasma glucose values were met or exceeded according to the IADPSG (International Association of et al., 2010): fasting glucose $\geq 5.1 \mathrm{mmol} / \mathrm{L}, 1 \mathrm{~h}$ glucose $\geq 10.0 \mathrm{mmol} / \mathrm{L}$, or $2 \mathrm{~h}$ glucose $\geq 8.5 \mathrm{mmol} / \mathrm{L}$.

\section{Perinatal outcomes}

Perinatal outcomes other than GDM were obtained from medical records, including gestational hypertension (GHT), preeclampsia (PE), hypothyroidism (HT), intrahepatic cholestasis of pregnancy (ICP), spontaneous preterm birth (sPTB). In addition, the information of gestational age at delivery, delivery mode, birth weight and NICU admission were also collected.

\section{Covariates}

Covariates were assessed using a structured questionnaire at the recruitment interview. We collected data on maternal age, ethnicity (Han Chinese, others), education level (junior secondary school or below, senior/technical secondary school, university or above), smoking status before pregnancy, parity $(0, \geq 1)$, chorionicity (monochorionicdiamniotic, dichorionic-diamniotic), mode of conception (naturally conceived, in vitro fertilization-embryo transfer), previous history of GDM, family history of GDM (first-degree relatives). Prepregnancy BMI $\left(\mathrm{kg} / \mathrm{m}^{2}\right)$ was calculated as the ratio of weight $(\mathrm{kg})$ to squared height $\left(\mathrm{m}^{2}\right)$, which was calculated from self-reported prepregnancy weight and measured height.

\section{Statistical analyses}

Principal component analysis with orthogonal (varimax) rotation was used to derive dietary patterns. We standardized the consumption frequency of each food group according to the mean and standard deviation before the extraction of dietary patterns. Four dietary patterns formed by linear combinations of each food group were selected by an inspection of scree plots and the interpretability of the results. The factor loadings, also known as coefficients defining these linear combinations, reflect the correlations of food groups with the corresponding dietary pattern. Food groups with loadings $>0.2$ were used to describe each dietary pattern. We calculated factor scores for each dietary pattern by summing the consumption frequencies of each food group and multiplying the sum by the factor loadings for each participant, and we categorized participants into quartiles based on their dietary pattern scores for subsequent analyses.

Frequencies and percentages are used to describe the distributions of categorical variables and continuous variables are expressed as the means $\pm \mathrm{SD}$. Chi-square tests or Fisher's exact tests were used to compare categorical variables between groups, and the continuous variables among groups were compared by using student $t$ test. The logistic regression models were used to estimate the odds ratio (OR) and 95\% confidence interval (CI) for GDM related to dietary pattern quartiles. We used the lowest quartile of the dietary pattern score as a reference. Multivariate linear regression models were used to examine the association between dietary pattern scores and plasma glucose levels following the OGTT. We conducted crude and adjusted analyses using the following models: Model 1, the crude model (individual dietary pattern); Model 2, Model 1 plus other dietary patterns; and Model 3, Model 2 plus maternal age, ethnicity, prepregnancy BMI, education level, smoking status, parity, previous history of GDM and family history of diabetes mellitus (DM). We also examined potential effect modification by age and prepregnancy weight status by including multiplicative interaction terms in the models.

All analyses were performed with SPSS software version 22.0 (SPSS, Inc.). For all statistical analyses, a two-tailed $p$ value less than 0.05 was considered statistically significant.

\section{Results}

\section{Characteristics of the participants}

After excluding women pregnant with twins who had a miscarriage $(n=16)$, who experienced fetal death of one of the twins $(n=11)$, who had incomplete FFQ records $(n=9)$, and who had missing OGTT results $(n=79)$, a total of 324 women were available for analysis (Fig. 1). There were no significant differences in regard to age, ethnicity, prepregnancy BMI, mode of conception, chorionicity or parity between the women who were included and those who were excluded.

The incidence of GDM was $31.2 \%$ in this study population (101 out of 324 pregnant women). Table 1

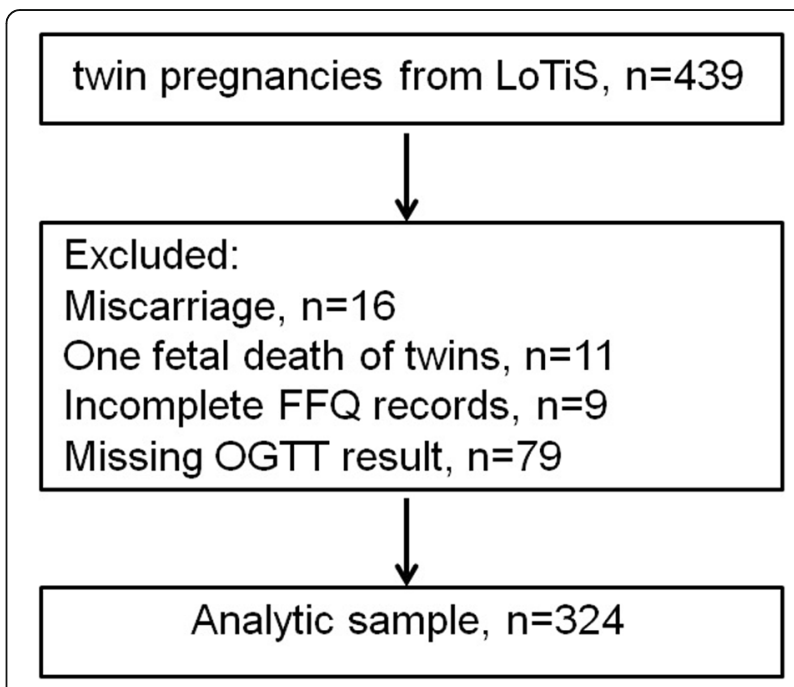

Fig. 1 Flowchart showing selection of participants included in this analysis from LoTiS study 
summarizes the participant characteristics according to GDM status. Overall, no significant differences were observed between GDM and non-GDM women in terms of ethnicity, education level, smoking status before pregnancy, chorionity, parity, mode of conception, previous history of GDM, family history of T2DM and energy intake. However, compared to women without GDM, women with GDM tended to be older ( $\geq 35$ years old) and were more likely to have a BMI higher than $24.0 \mathrm{~kg} /$ $\mathrm{m}^{2}$ before pregnancy (26.7\% versus $\left.17.5 \%\right)(p<0.01$ for both).

\section{Dietary pattern analysis}

In the present study, four main maternal dietary patterns accounted for $28.44 \%$ of the total variation. The factor loading for each dietary pattern are listed in Table 2 . The first pattern, named the "vegetable-based pattern", explained $9.24 \%$ of the total variance. This pattern was

Table 1 Characteristics of participants by GDM status ${ }^{a}$

\begin{tabular}{|c|c|c|c|c|}
\hline Variables & Overall $(n=324)$ & $\operatorname{GDM}(n=101)$ & Non-GDM $(n=223)$ & $P$-value ${ }^{b}$ \\
\hline Maternal age (y) & & & & 0.001 \\
\hline$<25$ & $36(11.1)$ & $3(3.0)$ & $33(14.8)$ & \\
\hline $25-29$ & $137(42.3)$ & $49(48.5)$ & $88(39.5)$ & \\
\hline $30-34$ & $113(34.9)$ & $30(29.7)$ & $83(37.2)$ & \\
\hline$\geq 35$ & $38(11.7)$ & 19 (18.8) & $19(8.5)$ & \\
\hline Pre-pregnancy BMI (kg/m²) & & & & 0.001 \\
\hline$<18.5$ & $39(12.0)$ & $3(3.0)$ & $36(16.1)$ & \\
\hline $18.5-23.9$ & $219(67.6)$ & $71(70.3)$ & $148(66.4)$ & \\
\hline$\geq 24.0$ & $66(20.4)$ & $27(26.7)$ & $39(17.5)$ & \\
\hline Ethnicity & & & & 0.743 \\
\hline Han Chinese & $303(93.5)$ & $97(96.0)$ & $206(92.4)$ & \\
\hline Others & $21(6.5)$ & $4(4.0)$ & $17(7.6)$ & \\
\hline Education level & & & & 0.486 \\
\hline Junior secondary school or below & $52(16.0)$ & $14(13.9)$ & $38(17.0)$ & \\
\hline Senior/technical secondary school & $36(11.1)$ & $14(13.9)$ & $22(9.9)$ & \\
\hline University or above & $236(72.8)$ & $73(72.3)$ & $163(73.1)$ & \\
\hline Smoking before pregnancy & & & & 0.872 \\
\hline No & $307(94.8)$ & $96(95.0)$ & $211(94.65)$ & \\
\hline Yes & $17(5.2)$ & $5(5.0)$ & $12(5.4)$ & \\
\hline Mode of conception & & & & 0.122 \\
\hline Natural conceived & $194(59.9)$ & $51(55.0)$ & $143(64.1)$ & \\
\hline In vitro fertilization-embryo transfer & $130(40.1)$ & $50(45.0)$ & $80(35.9)$ & \\
\hline Chorionicity & & & & 0.815 \\
\hline Monochorionic-diamniotic & $136(42.0)$ & $40(45.0)$ & $96(43.0)$ & \\
\hline Dichorionic-diamniotic & $188(58.0)$ & $61(55.0)$ & $127(57.0)$ & \\
\hline Parity & & & & 0.586 \\
\hline 0 & $239(73.8)$ & $77(76.2)$ & $162(72.6)$ & \\
\hline$\geq 1$ & $85(26.2)$ & $24(23.8)$ & $61(27.4)$ & \\
\hline Previous history of GDM & & & & 1.000 \\
\hline No & $319(98.5)$ & $99(98.0)$ & $220(98.7)$ & \\
\hline Yes & $5(1.5)$ & $2(2.0)$ & $3(1.3)$ & \\
\hline Family history of T2DM & & & & 0.493 \\
\hline No & $309(95.4)$ & $98(97.0)$ & $211(94.6)$ & \\
\hline Yes & $15(4.6)$ & $3(3.0)$ & $12(5.4)$ & \\
\hline
\end{tabular}

BMI Body mass index, GDM Gestational diabetes mellitus, DM Diabetes mellitus

${ }^{a}$ Numbers are presented as $\mathrm{n}(\%)$

${ }^{b}$ Based on $x^{2}$ tests 
Table 2 Factor loadings for the four dietary patterns identified from principal components analysis ${ }^{\mathrm{a}}$

\begin{tabular}{|c|c|c|c|}
\hline Dietary pattern & Food & Factor loading coefficient & Cumulative variance explained \\
\hline \multirow[t]{9}{*}{ Vegetable-based pattern } & Root vegetables & 0.723 & 9.24 \\
\hline & Gourd/melon family vegetables & 0.686 & \\
\hline & Freshwater fish & 0.491 & \\
\hline & Leafy and cruciferous vegetables & 0.488 & \\
\hline & Red meat & 0.471 & \\
\hline & Fried breads & 0.370 & \\
\hline & White rice & 0.301 & \\
\hline & Deep-sea fish and seafood products & 0.268 & \\
\hline & Dumplings & -0.222 & \\
\hline \multirow[t]{12}{*}{ Poultry-fruit-based pattern } & Poultry & 0.544 & 16.64 \\
\hline & Fresh fruit & 0.505 & \\
\hline & Processed fruit & 0.447 & \\
\hline & Soups & 0.424 & \\
\hline & Meat Innards & 0.422 & \\
\hline & Noodles & 0.373 & \\
\hline & Cereals & 0.347 & \\
\hline & Dairy products & 0.346 & \\
\hline & Deep-sea fish and seafood products & 0.322 & \\
\hline & Nuts/seeds & 0.319 & \\
\hline & Eggs & 0.315 & \\
\hline & White rice & -0.267 & \\
\hline \multirow[t]{9}{*}{ Sweets -based pattern } & Biscuits\pastries\cakes & 0.560 & 22.61 \\
\hline & Bread & 0.455 & \\
\hline & Deep-sea fish and seafood products & 0.314 & \\
\hline & Ethnic breads & 0.313 & \\
\hline & Dessert soup & 0.307 & \\
\hline & Dairy products & 0.296 & \\
\hline & Noodles & -0.436 & \\
\hline & Meat products & -0.405 & \\
\hline & Dumplings & -0.267 & \\
\hline \multirow[t]{8}{*}{ Plant protein-rich--based pattern } & Soya milk & 0.670 & 28.44 \\
\hline & Legumes & 0.644 & \\
\hline & Beans/bean products & 0.535 & \\
\hline & Bun & 0.442 & \\
\hline & Rice & 0.421 & \\
\hline & Bread & 0.407 & \\
\hline & Ethnic breads & 0.236 & \\
\hline & Cereals & -0.306 & \\
\hline
\end{tabular}

${ }^{\mathrm{a}}$ Factor loading $< \pm 0.200$ were not listed in the table for simplicity

characterized by a high intake of root vegetables, gourd/ melon family vegetables, freshwater fish, leafy and cruciferous vegetables, and red meat. The second pattern, named the "poultry-and-fruit-based pattern", explained $7.40 \%$ of the total variance. This pattern was characterized by a high intake of poultry, fresh fruit, processed fruit, soups and meat innards. The third pattern, named the "sweet-based pattern", explained 5.97\% of the total variance. This pattern was characterized by a high intake of biscuits, pastries, cakes, bread and deep- 
sea fish and seafood products. The fourth pattern, named the "plant-protein-based pattern", explained $5.83 \%$ of the total variance. This pattern was characterized by soya milk, legumes, beans or bean products, buns and rice.

Table 3 describes the participants' characteristics and their dairy energy consumption according to the quartiles of dietary pattern scores. Regarding the vegetable-based pattern, women with the highest score tended to be highly educated, were more likely to have monochorionicdiamniotic twin pregnancies and had higher intakes of total energy than those with the lowest score. For the poultry-and-fruit-based pattern, women with the highest score were more likely to have a IVF-ET mode of conception, and more likely to have dichorionic-diamniotic twin pregnancies than those with the lowest score. For the sweet-based pattern, women with the highest score had higher intakes of total energy and tended to have a higher incidence of GDM, but the GDM incidence was not significantly higher than that of those with the lowest score. Regarding the plant-protein-based pattern, women with the highest score had higher intakes of total energy than those with the lowest score.

Besides, the analyses of perinatal outcomes other than GDM according to the quartiles of dietary pattern scores was shown in Table 4. There were no correlations found between dietary patterns and the other pregnancy outcomes, except birth weight. The larger co-twin birth weight of women with the highest score in vegetablebased pattern is significantly lower than that of women with the lowest score in vegetable-based pattern. Similar trend has also been observed in the smaller co-twin, although statistical significance wasn't achieved. These facts indicate that women with vegetable-based pattern during the second trimester are more likely to deliver lighter offspring.

\section{Dietary patterns and risk of gestational diabetes mellitus}

Table 5 summarizes the univariate and multivariate regression analyses for the correlation between dietary pattern and risk of GDM. There was no significant correlation between any dietary pattern and the risk of GDM. Compared with the lowest quartiles of the dietary pattern scores, the multivariable-adjusted ORs for the corresponding highest quartile of the vegetable-based, poultry-and-fruit-based, sweet-based and plant-proteinbased patterns were 1.23 (95\% CI: 0.57, 2.66, $p>0.05$ ), 0.96 (95\% CI: $0.45,2.03, p>0.05), 1.97$ (95\% CI: 0.94 , 4.12, $p>0.05$ ) and 1.02 (95\% CI: 0.49, 2.09, $p>0.05$ ), respectively.

In the subgroup analyses (Fig. 2), a significant increase in GDM risk was observed only among nonoverweight women (prepregnancy $\mathrm{BMI}<24.0$ ) when comparing the highest quartile of sweet-based pattern scores to the lowest quartile (OR 2.69; 95\% CI: 1.09, 6.66; $p<0.05$ ), despite the lack of significance for the interaction between prepregnancy BMI and sweets-based pattern score $(\mathrm{p}$ for interaction $=0.267$ ). There was no effect modification by prepregnancy BMI on the association between other dietary patterns and GDM risk. There were no modification effects of any dietary patterns by age.

Furthermore, we examined the association of the sweet-based pattern with blood glucose levels following the OGTT, and the results showed that the sweet-based pattern was positively correlated with $1 \mathrm{~h}$ postload blood glucose among nonoverweight women $(\beta 0.18 ; 95 \% \mathrm{CI}$ : $0.01,0.35 ; p<0.05$ ) (Table 6).

\section{Discussion}

In this Chinese prospective twin pregnancies birth cohort study, four dietary patterns, namely, the vegetablebased pattern, the poultry-and-fruit-based pattern, the sweet-based pattern and the plant-protein-based pattern, were identified in the second trimester. No significant association was found between the four dietary patterns and the risk of GDM. However, we observed that the sweet-based pattern was significantly associated with an increased risk of GDM and higher blood glucose levels $1 \mathrm{~h}$ after the OGTT only among nonoverweight women. It is speculated that dietary intake might have little influence on prepregnancy nonoverweight women but not on prepregnancy overweight women.

In recent years, the impact of food intake on the risk of GDM has gained increasing attention. Dietary pattern analysis is a holistic approach to account for food consumption in a typical diet and take the synergy of food and nutrient intake into account. The majority of studies on dietary patterns and GDM risk were first conducted in Western populations. Generally, these studies found that a prudent dietary pattern that was high in seafood, eggs, vegetables, fruits, berries and vegetable oils [22], a prudent diet that was high in fruits, green vegetables and fish [23], and a 'Mediterranean' dietary pattern [24] were associated with a lower risk of GDM, while a 'Western' dietary pattern that was high in red and processed meat, French fries, pizza, sweets and desserts was positively associated with a higher risk of GDM [23]. Since the heterogeneity of dietary structure among the different countries or regions results in different dietary patterns, we paid special attention to studies that were conducted in the Chinese population [25-29]. In general, a western pattern high in dairy products and baked/fried food, and a sweet pattern high in cantonese desserts and sugarsweetened beverages were related to an increased risk of GDM, which were similar with the findings in a Western population [30], whereas a vegetable pattern rich in root 
Table 3 Characteristics and dairy energy consumption of participants by quartiles of dietary pattern scores ${ }^{a}$

\begin{tabular}{|c|c|c|c|c|c|c|c|c|}
\hline \multirow[t]{2}{*}{ Variables } & \multicolumn{2}{|c|}{ Vegetable } & \multicolumn{2}{|c|}{ Poultry-fruit } & \multicolumn{2}{|l|}{ Sweets } & \multicolumn{2}{|c|}{ Plant protein-rich } \\
\hline & Q1 & Q4 & Q1 & Q4 & Q1 & Q4 & Q1 & Q4 \\
\hline \multicolumn{9}{|l|}{ GDM } \\
\hline No & 59(72.8) & $60(74.1)$ & 58(71.6) & $54(66.7)$ & $61(75.3)$ & $49(60.5)$ & $55(67.9)$ & $54(66.7)$ \\
\hline Yes & $22(27.2)$ & $21(25.9)$ & $23(28.4)$ & 27(33.3) & $20(24.7)$ & $32(39.5)$ & $26(32.1)$ & $27(33.3)$ \\
\hline$P^{b}$ & 1.000 & & 0.610 & & 0.064 & & 1.000 & \\
\hline \multicolumn{9}{|l|}{ Maternal age (y) } \\
\hline$<25$ & $9(11.1)$ & $6(7.4)$ & 10(12.3) & $7(8.6)$ & $9(11.1)$ & $9(11.1)$ & $13(16.1)$ & $5(6.2)$ \\
\hline $25-29$ & 27(33.3) & $33(40.7)$ & $35(43.2)$ & $32(39.5)$ & $31(38.3)$ & $37(45.7)$ & $35(43.2)$ & $33(40.7)$ \\
\hline $30-34$ & $37(45.7)$ & $32(39.6)$ & 28(34.6) & $34(42.0)$ & $35(43.2)$ & $26(32.1)$ & $26(32.1)$ & $35(43.2)$ \\
\hline$\geq 35$ & $8(9.9)$ & 10(12.3) & $8(9.9)$ & $8(9.9)$ & $6(7.4)$ & $9(11.1)$ & $7(8.6)$ & $8(9.9)$ \\
\hline$P^{b}$ & 0.634 & & 0.757 & & 0.491 & & 0.172 & \\
\hline \multicolumn{9}{|l|}{ Pre-pregnancy BMI $\left(\mathrm{kg} / \mathrm{m}^{2}\right)$} \\
\hline$<18.5$ & $6(7.4)$ & $13(16)$ & 10(12.3) & 11(13.6) & $9(11.1)$ & $9(11.1)$ & $12(14.8)$ & $10(12.3)$ \\
\hline $18.5-23.9$ & 58(71.6) & $55(68)$ & $52(64.2)$ & $56(69.1)$ & $56(69.1)$ & 59(72.8) & $53(65.4)$ & $54(66.7)$ \\
\hline$\geq 24.0$ & $17(21)$ & $13(16)$ & 19(23.5) & 14(19.8) & 16(19.8) & 13(16.1) & 16(19.8) & $17(21)$ \\
\hline$P$ & 0.206 & & 0.664 & & 0.825 & & 0.944 & \\
\hline \multicolumn{9}{|l|}{ Ethnicity } \\
\hline Han Chinese & 72(88.9) & 79(97.5) & 79(97.5) & 76(93.8) & 74(91.4) & 75(92.6) & $72(88.9)$ & $79(97.5)$ \\
\hline Others & $9(11.1)$ & $2(2.5)$ & $2(2.5)$ & $5(6.2)$ & $7(8.6)$ & $6(7.4)$ & $9(11.1)$ & $2(2.5)$ \\
\hline$p^{b}$ & 0.057 & & 0.443 & & 1.000 & & 0.057 & \\
\hline \multicolumn{9}{|l|}{ Education level } \\
\hline Junior secondary school or below & $13(16)$ & 11(13.6) & 15(18.5) & 11(13.6) & 15(18.5) & $8(9.9)$ & 16(19.8) & $13(16)$ \\
\hline Senior/technical secondary school & 12(14.8) & $3(3.7)$ & $7(8.6)$ & 12(14.8) & 12(14.8) & $7(8.6)$ & $10(12.3)$ & $7(8.7)$ \\
\hline University or above & $56(69.1)$ & $67(82.7)$ & $59(72.9)$ & $58(71.6)$ & $54(66.7)$ & $66(81.5)$ & $55(67.9)$ & $61(75.3)$ \\
\hline$P^{b}$ & 0.035 & & 0.346 & & 0.113 & & 0.587 & \\
\hline \multicolumn{9}{|l|}{ Smoking before pregnancy } \\
\hline No & 75(92.6) & 76(93.8) & $77(95.1)$ & $77(95.1)$ & 79(97.5) & $77(95.1)$ & $76(93.8)$ & $78(96.3)$ \\
\hline Yes & $6(7.4)$ & $5(6.2)$ & $4(4.9)$ & $4(4.9)$ & $2(2.5)$ & $4(4.9)$ & $5(6.2)$ & $3(3.7)$ \\
\hline$P^{b}$ & 1.000 & & 1.000 & & 0.682 & & 0.720 & \\
\hline \multicolumn{9}{|l|}{ Mode of conception } \\
\hline Natural conceived & 43(53.1) & $54(66.7)$ & $62(76.5)$ & $39(48.1)$ & $50(61.7)$ & $40(49.4)$ & $44(54.3)$ & $44(54.3)$ \\
\hline IVF-ET & $38(46.9)$ & 27(33.3) & 19(23.5) & $42(51.9)$ & $31(38.3)$ & $41(50.6)$ & $37(45.7)$ & $37(45.7)$ \\
\hline$P^{b}$ & 0.109 & & $<0.001$ & & 0.155 & & 1.000 & \\
\hline \multicolumn{9}{|l|}{ Chorionicity } \\
\hline Monochorionic-diamniotic & 23(28.4) & $40(49.4)$ & $40(49.4)$ & 27(33.3) & $33(40.7)$ & $33(40.7)$ & $33(40.7)$ & $31(38.3)$ \\
\hline Dichorionic-diamniotic & 58(71.6) & $41(50.6)$ & $41(50.6)$ & $54(66.7)$ & $48(59.3)$ & 48(59.3) & $48(59.3)$ & $50(61.7)$ \\
\hline$p^{b}$ & 0.010 & & 0.055 & & 1.000 & & 0.872 & \\
\hline \multicolumn{9}{|l|}{ Parity } \\
\hline 0 & 58(71.6) & $60(74.1)$ & 58(71.6) & $65(80.2)$ & 59(72.8) & $67(82.7)$ & $61(75.3)$ & $61(75.3)$ \\
\hline$\geq 1$ & $23(28.4)$ & $21(25.9)$ & $23(28.4)$ & 16(19.8) & $22(27.2)$ & 14(17.3) & $20(24.7)$ & $20(24.7)$ \\
\hline$p^{b}$ & 0.860 & & 0.270 & & 0.185 & & 1.000 & \\
\hline \multicolumn{9}{|l|}{ Previous history of GDM } \\
\hline No & $81(100)$ & $80(98.8)$ & 80(98.8) & 79(97.5) & 80(98.8) & 79(97.5) & $81(100)$ & $79(97.5)$ \\
\hline Yes & $0(0)$ & $1(1.2)$ & $1(1.2)$ & $2(2.5)$ & $1(1.2)$ & $2(2.5)$ & $0(0)$ & $2(2.5)$ \\
\hline
\end{tabular}


Table 3 Characteristics and dairy energy consumption of participants by quartiles of dietary pattern scores ${ }^{\mathrm{a}}$ (Continued)

\begin{tabular}{|c|c|c|c|c|c|c|c|c|}
\hline \multirow[t]{2}{*}{ Variables } & \multicolumn{2}{|c|}{ Vegetable } & \multicolumn{2}{|c|}{ Poultry-fruit } & \multicolumn{2}{|l|}{ Sweets } & \multicolumn{2}{|c|}{ Plant protein-rich } \\
\hline & Q1 & Q4 & Q1 & Q4 & Q1 & Q4 & $\overline{\mathrm{Q} 1}$ & Q4 \\
\hline$p^{b}$ & 1.000 & & 1.000 & & 1.000 & & 1.000 & \\
\hline \multicolumn{9}{|c|}{ Family history of DM } \\
\hline No & 78(96.3) & $77(95.1)$ & 79(97.5) & 78(96.3) & 76(93.8) & 79(97.5) & $77(95.1)$ & 76(93.8) \\
\hline Yes & $3(3.7)$ & $4(4.9)$ & $2(2.5)$ & $3(3.7)$ & $5(6.2)$ & $2(2.5)$ & $4(4.9)$ & $5(6.2)$ \\
\hline$p^{b}$ & 1.000 & & 1.000 & & 0.443 & & 1.000 & \\
\hline \multicolumn{9}{|c|}{ Energy intake, $\mathrm{kcal} / \mathrm{d}$} \\
\hline$\geq 2100$ & $17(21)$ & $44(54.3)$ & $12(14.8)$ & $21(25.9)$ & 25(30.9) & $45(55.6)$ & $27(33.3)$ & $47(58)$ \\
\hline$<2100$ & $64(79)$ & $37(45.7)$ & 69(85.2) & $60(74.1)$ & $56(69.1)$ & $36(44.4)$ & $54(66.7)$ & $34(42)$ \\
\hline$p^{\mathrm{b}}$ & $<0.001$ & & 0.118 & & 0.002 & & 0.003 & \\
\hline
\end{tabular}

BMI Body mass index, GDM Gestational diabetes mellitus, DM Diabetes mellitus

${ }^{a}$ Numbers are presented as $\mathrm{n}(\%)$

${ }^{b}$ Based on $X^{2}$ tests

vegetables, beans and melon vegetables was related with a decreased risk of GDM. Due to the diversity of dietary traditions across China, the definition of 'Chinese traditional' diet pattern was not uniformed, and therefore, results in conflicting conclusions. For instance, a 'traditional pattern' that was high in vegetables, fruits, and rice was associated with a decreased risk of GDM [27, 29], whereas a 'traditional pattern' defined as the high intake of vegetables, fine grains, red meat and tubers was associated with an increased risk of GDM [26], the researchers in this study suggested that the increased likelihood of GDM may result from the effect of red meat.

Unlike prior research in the context of singleton pregnancies, the present study found no significant correlations between the identified dietary patterns and the risk of GDM in women pregnant with twins. Since previous studies have reported that associations between dietary patterns and GDM may vary by maternal characteristics, such as maternal age [29], prepregnancy BMI [22, 28], and maternal family history of diabetes [25], we examined potential effect modification by age and prepregnancy weight status. A significant association was observed between high sweet food intake and the risk of GDM among non-overweight women, whereas no association was found among overweight women. Additionally, we found that high sweet food intake influenced 1-h blood glucose levels following the OGTT among nonoverweight women. There were no modification effects of any dietary patterns by age. One possible reason may be due to the high incidence of GDM in this study (31.2\%). The environmental exposure factors had limited influence on the blood glucose levels, which was consistent with our previous result showing that there was no correlation between gestational weight gain and the incidence of GDM [31]. An alternative explanation was that there may be differences between twin and singleton gestation in terms of the development of GDM. A study showed that the mean serum concentration of human placental lactogen (hPL) at 30 and 36 weeks of gestation was markedly elevated in twin pregnancies compared with the concentration in singleton pregnancies. Higher levels of hormones, such as hPL, estrogen and progesterone, in twin pregnancies may influence the frequency of GDM through their insulin antagonistic effects [32]. In addition, another study suggested that placental mass and the number of fetuses contribute to the occurrence of GDM [33]. These reports support the hypothesis that increasing placental mass and increasing diabetogenic hormones may play an important role in the etiology of GDM in twin pregnancies.

We did not detect an effect modification by maternal family history of diabetes since only 15 (4.6\%) participants had a family history of T2DM. The rate of women with a family history of T2DM in our study appeared to be very low, but we could explain it. An epidemiological study of DM has shown that the prevalence of DM was $11.6-13.2 \%$ in Chongqing region, China [34]. On the other hand, a similar dietary pattern study performed in an adjacent area in western China revealed that $6.2 \%$ of pregnant women out of 1337 participants had a family history of T2D, and this rate is similar to that of our study [28]. Together with the relatively small sample size of this study, these factors lead to the low incidence of a family history of diabetes.

The strength of our study is the dietary patterns we identified reflected the habitual diet in the second trimester. Most pregnant women had a poor appetite or unusual tastes in the first trimester due to the gestational reactions of nausea and vomiting, and the dietary intake during this period has limited research significance. In the second trimester, pregnant women had a 
Table 4 Comparison of other pregnancy outcomes between quartiles of dietary pattern scores ${ }^{a}$

\begin{tabular}{|c|c|c|c|c|c|c|c|c|}
\hline \multirow[t]{2}{*}{ Variables } & \multicolumn{2}{|l|}{ Vegetable } & \multicolumn{2}{|l|}{ Poultry-fruit } & \multicolumn{2}{|l|}{ Sweets } & \multicolumn{2}{|c|}{ Plant protein-rich } \\
\hline & Q1 & Q4 & Q1 & Q4 & Q1 & Q4 & Q1 & Q4 \\
\hline \multicolumn{9}{|l|}{ GHP } \\
\hline No & 78(96.3) & 75(92.6) & 80(98.8) & 77(95.1) & 79(97.5) & 75(92.6) & 78(96.3) & 78(96.3) \\
\hline Yes & $3(3.7)$ & $6(7.4)$ & $1(1.2)$ & $4(4.9)$ & $2(2.5)$ & $6(7.4)$ & $3(3.7)$ & $3(3.7)$ \\
\hline$p^{b}$ & 0.495 & & 0.367 & & 0.277 & & 1.000 & \\
\hline \multicolumn{9}{|l|}{ PE } \\
\hline No & 77(95.1) & 74(91.4) & 77(95.1) & 76(93.8) & 77(95.1) & 76(93.8) & 79(97.5) & 75(92.6) \\
\hline Yes & $4(4.9)$ & $7(8.6)$ & $4(4.9)$ & $5(6.2)$ & $4(4.9)$ & $5(6.2)$ & $2(2.5)$ & $6(7.4)$ \\
\hline$p^{b}$ & 0.540 & & 1.000 & & 1.000 & & 0.277 & \\
\hline \multicolumn{9}{|l|}{ HT } \\
\hline No & 79(97.5) & 78(96.3) & $77(95.1)$ & 78(96.3) & 78(96.3) & $77(95.1)$ & 76(93.8) & $77(95.1)$ \\
\hline Yes & $2(2.5)$ & $3(3.7)$ & $4(4.9)$ & $3(3.7)$ & $3(3.7)$ & $4(4.9)$ & $5(6.2)$ & $4(4.9)$ \\
\hline$P^{b}$ & 1.000 & & 1.000 & & 1.000 & & 1.000 & \\
\hline \multicolumn{9}{|l|}{ ICP } \\
\hline No & 77(95.1) & 70(86.4) & 72(88.9) & 70(86.4) & 70(86.4) & $71(87.7)$ & $69(85.2)$ & $71(87.7)$ \\
\hline Yes & $4(4.9)$ & 11(13.6) & $9(11.1)$ & 11(13.6) & 11(13.6) & 10(12.3) & 12(14.8) & $10(12.3)$ \\
\hline$p^{b}$ & 0.058 & & 0.812 & & 1.000 & & 0.819 & \\
\hline GA (wk) & $36.6 \pm 1.5$ & $36.3 \pm 1.6$ & $36.5 \pm 1.5$ & $36.3 \pm 1.6$ & $36.4 \pm 1.7$ & $36.3 \pm 1.4$ & $36.3 \pm 1.6$ & $36.6 \pm 1.1$ \\
\hline$P^{c}$ & 0.242 & & 0.399 & & 0.772 & & 0.132 & \\
\hline \multicolumn{9}{|l|}{ sPTB } \\
\hline No & $60(74.1)$ & 63(77.8) & $66(81.5)$ & $62(76.5)$ & $65(80.2)$ & $55(67.9)$ & 64(79.0) & 64(79.0) \\
\hline Yes & $21(25.9)$ & $18(22.2)$ & 15(18.5) & $19(23.5)$ & 16(19.8) & $26(32.1)$ & $17(21.0)$ & 17(21.0) \\
\hline$p^{b}$ & 0.714 & & 0.563 & & 0.106 & & 1.000 & \\
\hline \multicolumn{9}{|l|}{ Cesarean } \\
\hline No & $0(0.0)$ & $1(1.2)$ & $2(2.5)$ & $1(1.2)$ & $2(2.5)$ & $0(0.0)$ & $0(0.0)$ & $0(0.0)$ \\
\hline Yes & $81(100)$ & $80(98.8)$ & 79(97.5) & 80(98.8) & 79(97.5) & $81(100)$ & $81(100)$ & $81(100)$ \\
\hline$P^{b}$ & 1.000 & & 1.000 & & 0.497 & & 1.000 & \\
\hline$B W^{H}(g)$ & $2675 \pm 424$ & $2525 \pm 408$ & $2628 \pm 413$ & $2649 \pm 408$ & $2585 \pm 424$ & $2637 \pm 360$ & $2602 \pm 425$ & $2690 \pm 388$ \\
\hline$p^{c}$ & 0.024 & & 0.750 & & 0.394 & & 0.174 & \\
\hline$B W^{L}(g)$ & $2389 \pm 434$ & $2262 \pm 412$ & $2354 \pm 391$ & $2368 \pm 411$ & $2311 \pm 431$ & $2380 \pm 392$ & $2323 \pm 456$ & $2383 \pm 401$ \\
\hline$p^{c}$ & 0.059 & & 0.827 & & 0.284 & & 0.377 & \\
\hline \multicolumn{9}{|l|}{$\mathrm{NICU}$} \\
\hline No & 68(84.0) & $61(75.3)$ & 63(77.8) & $65(80.2)$ & $65(80.2)$ & 63(77.8) & $66(81.5)$ & 68(84.0) \\
\hline Yes & 13(16.0) & $20(24.7)$ & 18(22.2) & 16(19.8) & 16(19.8) & $18(22.2)$ & 15(18.5) & 13(16.0) \\
\hline$p^{b}$ & 0.242 & & 0.847 & & 0.847 & & 0.836 & \\
\hline
\end{tabular}

GHP Gestational hypertension, PE Preeclampsia, HT Hypothyroidism, ICP Intrahepatic cholestasis of pregnancy, GA Gestational age, sPTB Spontaneous preterm birth, $B W^{H}$ Heavier twin birthweight, $B W^{L}$ Lighter twin birthweight

${ }^{a}$ Numbers are presented as $n(\%)$ or means \pm SD

${ }^{b}$ Based on $x^{2}$ tests or Fisher exact test

c Based on student $t$ test

better appetite, and we used the FFQ to investigate their dietary intake, which has the advantage of capturing long-term habitual diet [35], additionally, GDM was diagnosed in this period. It is reasonable to explore the influence of dietary intake on the risk of GDM. Another strength of our study was the specific study population.
This is the first study using a population pregnant with twins to explore the effects of dietary patterns on the risk of GDM.

This study contributes new knowledge regarding the relationship between dietary intake and risk of GDM in twin pregnancies, but several limitations of this study should be 
Table 5 Logistic regression analysis for the risk of GDM according to the quartiles of dietary pattern scores

\begin{tabular}{|c|c|c|c|c|c|}
\hline \multirow{2}{*}{$\begin{array}{l}\text { Dietary } \\
\text { patterns }\end{array}$} & \multirow{2}{*}{$\begin{array}{l}\text { Q1 }(n=81) \\
\text { Reference }\end{array}$} & Q2 $(n=81)$ & \multirow[t]{2}{*}{ Q3 $(n=81)$} & \multirow[t]{2}{*}{$\mathrm{Q} 4(n=81)$} & \multirow{2}{*}{$\begin{array}{l}P \text { for } \\
\text { trend }\end{array}$} \\
\hline & & Odds Ratio (OR) (95\% Cl) & & & \\
\hline \multicolumn{6}{|c|}{ Vegetable-based pattern } \\
\hline Model $1^{\mathrm{a}}$ & 1.00 & $1.20(0.61-2.36)$ & $1.14(0.65-2.57)$ & $0.94(0.47-1.89)$ & 0.169 \\
\hline Model $2^{\mathrm{b}}$ & 1.00 & $1.18(0.59-2.36)$ & $1.12(0.61-2.24)$ & $0.98(0.47-2.01)$ & 0.235 \\
\hline Model $3^{c}$ & 1.00 & $1.50(0.72-3.14)$ & $1.89(0.90-3.98)$ & $1.23(0.57-2.66)$ & 0.357 \\
\hline \multicolumn{6}{|c|}{ Poultry-fruit-based pattern } \\
\hline Model $1^{a}$ & 1.00 & $1.12(0.58-2.19)$ & $1.00(0.51-1.96)$ & $1.19(0.60-2.31)$ & 0.943 \\
\hline Model $2^{b}$ & 1.00 & $1.10(0.55-2.18)$ & $1.05(0.51-2.16)$ & $1.10(0.54-2.20)$ & 0.994 \\
\hline Model $3^{c}$ & 1.00 & $1.02(0.50-2.11)$ & $0.98(0.46-2.10)$ & $0.96(0.45-2.03)$ & 0.999 \\
\hline \multicolumn{6}{|c|}{ Sweets-based pattern } \\
\hline Model $1^{a}$ & 1.00 & $1.36(0.68-2.72)$ & $1.28(0.64-2.57)$ & $1.99(1.02-3.91)$ & 0.236 \\
\hline Model $2^{b}$ & 1.00 & $1.24(0.61-2.52)$ & $1.17(0.57-2.38)$ & $1.84(0.92-3.69)$ & 0.340 \\
\hline Model $3^{c}$ & 1.00 & $1.37(0.65-2.89)$ & $1.34(0.64-2.83)$ & $1.97(0.94-4.12)$ & 0.349 \\
\hline \multicolumn{6}{|c|}{ Plant protein-rich-based pattern } \\
\hline Model $1^{\mathrm{a}}$ & 1.00 & $1.18(0.61-2.28)$ & $0.78(0.40-1.56)$ & $0.82(0.58-2.07)$ & 0.649 \\
\hline Model $2^{\mathrm{b}}$ & 1.00 & $1.13(0.58-2.22)$ & $0.75(0.37-1.54)$ & $0.81(0.59-1.92)$ & 0.655 \\
\hline Model $3^{c}$ & 1.00 & $0.98(0.48-2.00)$ & $0.68(0.32-1.43)$ & $1.02(0.49-2.09)$ & 0.667 \\
\hline
\end{tabular}

${ }^{\text {aCrude model }}$

${ }^{\mathrm{b}}$ Adjusted for other dietary patterns

'Model 2 plus maternal age, pre-pregnancy BMI, ethnicity, education level, parity, smoking status, chorionicity, mode of conception, previous history of GDM and family history of DM

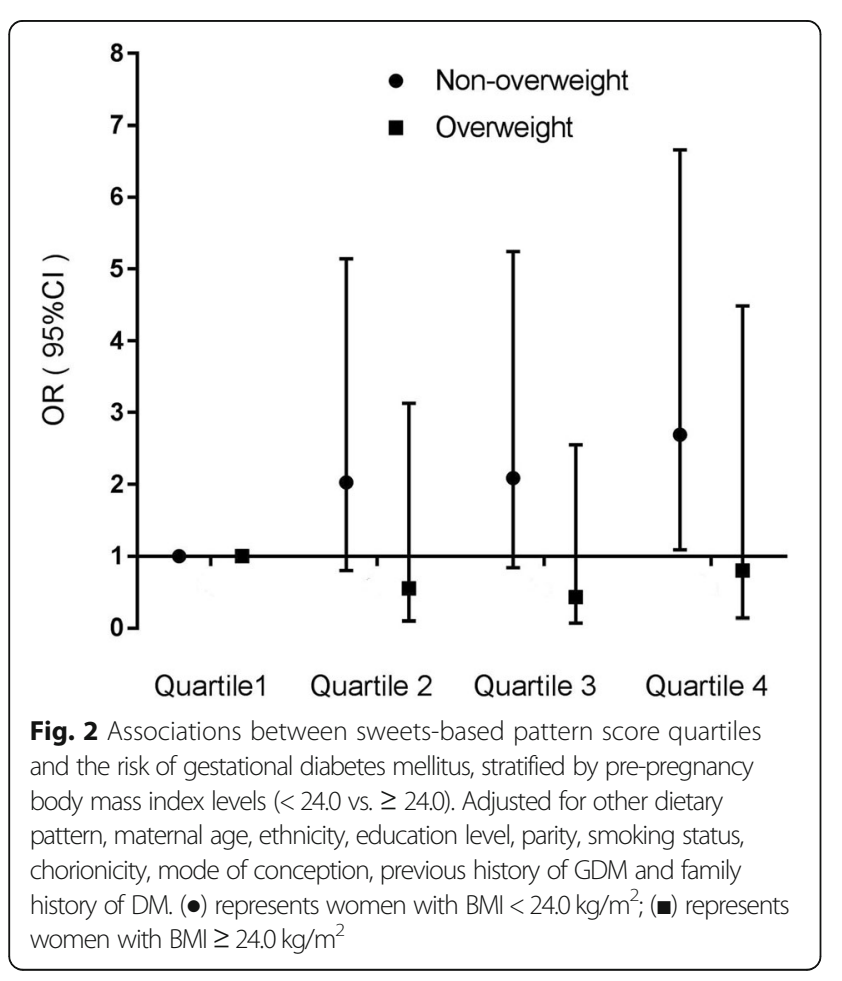

taken into consideration. Although it is a common sense that a prospective twin birth cohort is extremely difficult to establish, the relatively small sample size is a weakness of this study, as a sample size that is at least $5 \sim 10$ fold the number of questionnaire items is required to attain adequate statistical power. Additionally, although FFQs have the advantage of capturing long-term habitual dietary intake, they have a limited ability to accurately and prospectively recording food intake [35]. Finally, the lack of information related to glycemic control after GDM was diagnosed could be improved in future studies. Blood glucose level assessment would be expected as a follow-up study to observe the short-term and long-term influences of GDM on maternal and neonatal outcomes. This study found no relationship between the vegetable dietary pattern during the second trimester and the incidence of GDM however, this dietary pattern may affect the birth weight of offspring in the presence or absence of GDM. However, the maternal nutrition status during the third trimester also has profound impact on fetal birth weight, further trimester-based nutritional investigations are warranted to decipher the correlation between vegetable dietary pattern and fetal birth weight of twin pregnancy.

\section{Conclusion}

This is the first study to specifically investigate the effects of dietary patterns on the risk of GDM in a population pregnant with twins. Four dietary patterns were 
Table 6 Linear regression analysis of correlations between the sweet-based pattern and blood glucose levels following OGTT

\begin{tabular}{|c|c|c|c|c|c|c|}
\hline \multirow[t]{2}{*}{ Variables } & \multicolumn{6}{|c|}{ Blood Glucose Level $\beta$ (95\% Cl) } \\
\hline & Fasting & $p$-value & $1 \mathrm{~h}$ after OGTT & $p$-value & $2 \mathrm{~h}$ after OGTT & $p$-value \\
\hline \multicolumn{7}{|c|}{ Non-overweight } \\
\hline Model $1^{\mathrm{a}}$ & $0.03(-0.0-0.07)$ & 0.117 & $0.20(0.03-0.37)$ & 0.020 & $0.12(-0.02-0.26)$ & 0.091 \\
\hline Model $2^{b}$ & $0.03(-0.01-0.07)$ & 0.103 & $0.18(0.01-0.36)$ & 0.039 & $0.11(-0.04-0.25)$ & 0.148 \\
\hline Model $3^{c}$ & $0.03(-0.01,0.07)$ & 0.102 & $0.18(0.01-0.35)$ & 0.039 & $0.12(-0.02-0.26)$ & 0.103 \\
\hline \multicolumn{7}{|l|}{ Overweight } \\
\hline Model $1^{\mathrm{a}}$ & $-0.07(-0.17-0.03)$ & 0.181 & $-0.24(-0.63-0.15)$ & 0.228 & $-0.18(-0.48-0.13)$ & 0.250 \\
\hline Model $2^{b}$ & $-0.08(-0.18-0.03)$ & 0.145 & $-0.24(-0.62-0.15)$ & 0.218 & $-0.17(-0.47-0.14)$ & 0.287 \\
\hline Model $3^{c}$ & $-0.08(-0.17-0.02)$ & 0.133 & $-0.23(-0.61-0.16)$ & 0.248 & $-0.17(-0.48-0.14)$ & 0.275 \\
\hline
\end{tabular}

${ }^{a}$ Crude model

${ }^{\mathrm{b}}$ Adjusted for other dietary patterns

${ }^{c}$ Model 2 plus maternal age, ethnicity, education level, parity, smoking status, chorionicity, mode of conception, previous history of GDM and family history of DM

identified: a vegetable-based pattern, a poultry-and-fruitbased pattern, a sweet-based pattern and a plantprotein-based pattern. Although our study indicated that no dietary patterns were associated with the risk of GDM in twin pregnancies, there was a significant positive association between the sweet-based diet pattern characterized by a high intake of biscuits $\backslash$ pastrieslcakes, breads, desserts and the incidence of GDM among women pregnant with twins who were not overweight prior to pregnancy. Further research is needed to elucidate the role of glucose levels in maternal and neonatal outcomes in Chinese women who are pregnant with twins.

\section{Abbreviations}

BMI: Body mass index; BW: Birth weight; Cl: Confidence interval; FFQ: Food frequency questionnaire; GA: Gestational age; GDM: Gestational diabetes mellitus; GHT: Gestational hypertension; hPL: Human placental lactogen; HT: Hypothyroidism; IADPSG: International Association of Diabetes and Pregnancy Study Groups; ICP: Intrahepatic cholestasis of pregnancy; LoTiS: Longitudinal Twin Study; NICU: Neonatal Intensive Care Unit; OGTT: Oral glucose tolerance test; OR: Odds ratio; PE: Preeclampsia; sPTB: Spontaneous preterm birth; T2DM: Type 2 Diabetes Mellitus

\section{Acknowledgments}

The authors would like to thank all the participants, health professionals and researchers who contributed to this cohort study.

\section{Authors' contributions}

LW, CT, HQ and PNB designed the research protocol; LW, HG, XC and YZ conducted the study; LW, JG and JY analysed the data; LW and CT drafted the manuscript; JQ, LZ, MDK and RS critically revised the manuscript; $C T$ and $\mathrm{HB}$ were responsible for the final contents. All authors read and approved the final manuscript.

\section{Funding}

This work was supported by the National Key Research and Development Program of China (2018YFC1002900), and National Natural Science Foundation of China $(81520108013,81771613,81901508)$. In addition, this study was supported by the "111 program" of the Ministry of Education P.R.C and the State Administration of Foreign Experts Affairs P.R.C., and Key Laboratory of Birth Defects and Reproductive Health of National Health and Family Planning Commission P.R.C.

\section{Availability of data and materials}

The datasets used and/or analyzed during the current study are available from the corresponding author upon request.
Ethics approval and consent to participate

This study was approved by the First Affiliated Hospital of Chongqing Medical University. All participants have signed a informed consent before recruitment to the study.

\section{Consent for publication}

Participants were provided a study overview and verbal consent was attained.

\section{Competing interests}

The authors declare that they have no competing interests.

\section{Author details}

${ }^{1}$ Department of Obstetrics, The First Affiliated Hospital of Chongqing Medical University, Chongqing 400016, China. ${ }^{2}$ International Collaborative Laboratory of Reproduction and Development, Ministry of Education, Chongqing Medical University, Chongqing 400016, China. ${ }^{3}$ State Key Laboratory of Maternal and Fetal Medicine of Chongqing Municipality, The First Affiliated Hospital of Chongqing Medical University, Chongqing 400016, China.

${ }^{4}$ Chengdu Women's and Children's Central Hospital, School of Medicine, University of Electronic Science and Technology of China, Chengdu 611731, China. ${ }^{5}$ Fetal Medicine Centre, Birmingham Women's \& Children's Foundation Trust, Birmingham B15 2TG, UK. Institute of Metabolism \& Systems Research, University of Birmingham, Birmingham B15 2TT, UK. ${ }^{7}$ Cancer, Disease and Developmental Epigenetics, Murdoch Children's Research Institute, and Department of Paediatrics University of Melbourne, Parkville, Victoria 3052, Australia. ${ }^{8}$ Fujian Provincial Maternity and Children's Hospital, Affiliated Hospital of Fujian Medical University, Fuzhou 350001, Fujian, China. ${ }^{9}$ College of Life Sciences, University of Leicester, Leicester LE1 $7 \mathrm{RH}, \mathrm{UK}$.

Received: 31 August 2019 Accepted: 30 January 2020

Published online: 10 February 2020

\section{References}

1. Expert Committee on the Diagnosis and Clasification of Diabetes Mellitus. American Diabetes Association Clinical Practice Recommendations 2001. Diabetes Care. 2001:24(Suppl 1):S1-133.

2. Crowther CA, Hiller JE, Moss JR, Australian Carbohydrate Intolerance Study in Pregnant Women Trial G, et al. Effect of treatment of gestational diabetes mellitus on pregnancy outcomes. N Engl J Med. 2005;352:2477-86.

3. International Association of D, Pregnancy Study Groups Consensus P, Metzger BE, et al. International association of diabetes and pregnancy study groups recommendations on the diagnosis and classification of hyperglycemia in pregnancy. Diabetes Care. 2010;33:676-82.

4. Wei $Y$, Yang $H$, Zhu W, et al. International association of diabetes and pregnancy study group criteria is suitable for gestational diabetes mellitus diagnosis: further evidence from China. Chin Med J (Engl). 2014;127:3553-6.

5. Landon MB, Rice MM, Varner MW, et al. Mild gestational diabetes mellitus and long-term child health. Diabetes Care. 2015;38:445-52. 
6. Song C, Lyu Y, Li C, et al. Long-term risk of diabetes in women at varying durations after gestational diabetes: a systematic review and meta-analysis with more than 2 million women. Obes Rev. 2018;19:421-9.

7. Bao W, Bowers K, Tobias DK, et al. Prepregnancy dietary protein intake, major dietary protein sources, and the risk of gestational diabetes mellitus: a prospective cohort study. Diabetes Care. 2013:36:2001-8.

8. Bo S, Menato G, Lezo A, et al. Dietary fat and gestational hyperglycaemia. Diabetologia. 2001;44:972-8.

9. Ley SH, Hanley AJ, Retnakaran R, et al. Effect of macronutrient intake during the second trimester on glucose metabolism later in pregnancy. Am J Clin Nutr. 2011;94:1232-40.

10. Park S, Kim MY, Baik SH, et al. Gestational diabetes is associated with high energy and saturated fat intakes and with low plasma visfatin and adiponectin levels independent of prepregnancy BMI. Eur J Clin Nutr. 2013; 67:196-201.

11. Wang $Y$, Storlien $L H$, Jenkins $A B$, et al. Dietary variables and glucose tolerance in pregnancy. Diabetes Care. 2000;23:460-4.

12. Radesky JS, Oken E, Rifas-Shiman SL, et al. Diet during early pregnancy and development of gestational diabetes. Paediatr Perinat Epidemiol. 2008:22:47-59.

13. Pham NM, Do W, Lee AH. Polyphenol-rich foods and risk of gestational diabetes: a systematic review and meta-analysis. Eur J Clin Nutr. 2019;73(5): 647-56

14. Schoenaker DA, Soedamah-Muthu SS, Callaway LK, et al. Pre-pregnancy dietary patterns and risk of gestational diabetes mellitus: results from an Australian population-based prospective cohort study. Diabetologia. 2015; 58:2726-35.

15. Shin D, Lee KW, Song WO. Dietary patterns during pregnancy are associated with risk of gestational diabetes mellitus. Nutrients. 2015;7:9369-82.

16. Yoo EH, Chun D, Kim MJ, et al. Comparison of perinatal outcomes in late preterm birth between singleton and twin pregnancies. Obstet Gynecol Sci. 2017:60:421-6.

17. Hiersch L, Berger H, Okby R, et al. Incidence and risk factors for gestational diabetes mellitus in twin versus singleton pregnancies. Arch Gynecol Obstet. 2018;298:579-87.

18. Retnakaran $\mathrm{R}$, Shah BR. Impact of twin gestation and fetal sex on maternal risk of diabetes during and after pregnancy. Diabetes Care. 2016:39:e110-1.

19. Guillen MA, Herranz L, Barquiel B, et al. Influence of gestational diabetes mellitus on neonatal weight outcome in twin pregnancies. Diabet Med. 2014;31:1651-6.

20. Tong C, Wen $L$, Xia $Y$, et al. Protocol for a longitudinal twin birth cohort study to unravel the complex interplay between early-life environmental and genetic risk factors in health and disease: the Chongqing Longitudinal Twin Study (LoTiS). BMJ Open. 2018;8:e017889.

21. Chen X, de Seymour JV, Han TL, et al. Metabolomic biomarkers and novel dietary factors associated with gestational diabetes in China. Metabolomics. 2018;14:149

22. Tryggvadottir EA, Medek $H$, Birgisdottir BE, et al. Association between healthy maternal dietary pattern and risk for gestational diabetes mellitus. Eur J Clin Nutr. 2016;70:237-42.

23. Zhang C, Schulze MB, Solomon CG, et al. A prospective study of dietary patterns, meat intake and the risk of gestational diabetes mellitus. Diabetologia. 2006;49:2604-13.

24. Karamanos B, Thanopoulou A, Anastasiou E, et al. Relation of the Mediterranean diet with the incidence of gestational diabetes. Eur J Clin Nutr. 2014:68:8-13.

25. He JR, Yuan MY, Chen NN, et al. Maternal dietary patterns and gestational diabetes mellitus: a large prospective cohort study in China. Br J Nutr. 2015; 113:1292-300

26. Du HY, Jiang $\mathrm{H}$, Karmin $\mathrm{O}$, et al. Association of dietary pattern during pregnancy and gestational diabetes mellitus: a prospective cohort study in northern China. Biomed Environ Sci. 2017:30:887-97.

27. Zhou X, Chen R, Zhong C, et al. Maternal dietary pattern characterised by high protein and low carbohydrate intake in pregnancy is associated with a higher risk of gestational diabetes mellitus in Chinese women: a prospective cohort study. Br J Nutr. 2018;120:1045-55.

28. Mak JKL, Pham NM, Lee AH, et al. Dietary patterns during pregnancy and risk of gestational diabetes: a prospective cohort study in Western China. Nutr J. 2018:17:107.

29. Hu J, Oken E, Aris IM, et al. Dietary patterns during pregnancy are associated with the risk of gestational diabetes mellitus: evidence from a
Chinese prospective birth cohort study. Nutrients. 2019;11. https://doi.org/ 10.3390/nu11020405.

30. Bartakova V, Kuricova K, Zlamal F, et al. Differences in food intake and genetic variability in taste receptors between Czech pregnant women with and without gestational diabetes mellitus. Eur J Nutr. 2018;57:513-21.

31. Wen $L$, Liu X, Wang $L$, et al. Correlation between second trimester weight gain and perinatal outcomes in dichorionic twin pregnancies: the LoTiS cohort study. Eur J Obstet Gynecol Reprod Biol. 2019;233:64-9.

32. Spellacy WN, Buhi WC, Birk SA. Human placental lactogen levels in multiple pregnancies. Obstet Gynecol. 1978;52:210-2.

33. Sivan E, Maman E, Homko CJ, et al. Impact of fetal reduction on the incidence of gestational diabetes. Obstet Gynecol. 2002;99:91-4

34. Zhou M, Astell-Burt T, Bi Y, et al. Geographical variation in diabetes prevalence and detection in China: multilevel spatial analysis of 98,058 adults. Diabetes Care. 2015:38:72-81.

35. Crozier SR, Inskip HM, Godfrey KM, et al. Dietary patterns in pregnant women: a comparison of food-frequency questionnaires and $4 \mathrm{~d}$ prospective diaries. Br J Nutr. 2008;99:869-75.

\section{Publisher's Note}

Springer Nature remains neutral with regard to jurisdictional claims in published maps and institutional affiliations.
Ready to submit your research? Choose BMC and benefit from:

- fast, convenient online submission

- thorough peer review by experienced researchers in your field

- rapid publication on acceptance

- support for research data, including large and complex data types

- gold Open Access which fosters wider collaboration and increased citations

- maximum visibility for your research: over $100 \mathrm{M}$ website views per year

At $\mathrm{BMC}$, research is always in progress.

Learn more biomedcentral.com/submissions 